Rev Esp Casos Clin Med Intern (RECCMI). 2020 (Dic); 5(3): 117-119

\title{
Miasis cutánea forunculoide
}

Reinaldo Luis Menéndez-Capote ${ }^{1}$, Hilario Olo-Olo ${ }^{2}$, Paulina Obono-Engang ${ }^{3}$, María de los Ángeles Chappotten-Delahanty ${ }^{4}$

${ }^{\prime}$ Servicio de Medicina Interna. Instituto de Medicina Tropical Pedro Kouri. La Habana. Cuba

${ }^{2}$ Servicio de Medicina Interna. Policlínico Virgen de Guadalupe. Ciudad de Mongomo. República de Guinea Ecuatorial

${ }^{3}$ Servicio de Infectología. Policlínico Virgen de Guadalupe. Mongomo Ciudad de Mongomo. República de Guinea Ecuatorial

${ }^{4}$ Servicio de Anatomía Patológica. Hospital clínico quirúrgico Joaquín Albarrán. La Habana. Cuba

Recibido: $31 / 07 / 2020$

Aceptado: 14/09/2020

En línea: 31/12/2020

Citar como: Menéndez-Capote RL, Olo-Olo H, Obono-Engang P, Chappotten-Delahanty MÁ. Miasis cutánea forunculoide. Rev Esp Casos Clin Med Intern (RECCMI). 2020 (Dic); 5(3): 117-119. doi: 10.32818/reccmi.a5n3a6.

Cite this as: Menéndez-Capote RL, Olo-Olo H, Obono-Engang P, Chappotten-Delahanty MÁ. Furunculoid cutaneous myiasis. Rev Esp Casos Clin Med Intern (RECCMI). 2020 (Dec); 5(3): 117-119. doi: 10.32818/reccmi.a5n3a6.

Autor para correspondencia: Reinaldo Luis Menéndez-Capote. maykelke@gmail.com

\section{Palabras clave \\ $\triangleright$ Miasis forunculoide \\ $\triangleright$ Cordylobia antropophaga \\ $\triangleright$ Dípteros \\ $\triangleright$ Larvas}

\section{Resumen}

La miasis es la infección de los tejidos del cuerpo humano o de animales por larvas de insectos del género dípteros. Se presenta un caso de miasis cutánea forunculoide (Cordylobia antropophaga) adquirida durante los primeros días de estancia en Guinea Ecuatorial con un número elevado de larvas, un estado de ansiedad importante y una rápida respuesta al tratamiento. Se describen sus características clínicas, su distribución geográfica y las opciones terapéuticas, con el objetivo de contribuir al conocimiento, sobre todo, de profesionales de países no endémicos.

Abstract
Myiasis is the infection of the human or animal body's tissues by insect larvae of the genus dipterous. We present
a case of furunculoid cutaneous myiasis (Cordylobia antropophaga) acquired during the first days of stay in
Guinea Ecuatorial with a high number of larvae, state of anxiety, and rapid response to treatment. We describe
its clinical characteristics, distribution, and therapeutic options to contribute to knowledge, especially, of pro-
fessionals from non-endemic countries.

\section{Puntos destacados}

$\triangleright$ Las miasis son frecuentes en países donde las condiciones higiénicas sanitarias son muy deficientes y existe el agente transmisor.

$\triangleright$ Constituyen una parte sustancial de los diagnósticos a los que se enfrentan los médicos. Su abordaje terapéutico es relativamente sencillo.

\section{Introducción}

La miasis, término propuesto por Hope en 1840, es la infección de los tejidos del cuerpo humano o de animales por larvas de insectos del género dípteros, que, durante un período de su desarrollo, se alimentan de tejidos vivos o muertos, o del alimento ingerido por el huésped ${ }^{1-3}$.

En su desarrollo están implicadas frecuentemente dos especies: la Dermatobia hominis, procedente de América Central y del Sur (mosca zumbadora) y la Cordylobia anthropophaga, ampliamente distribuida en el África Subsahariana (mosca Tumbu) ${ }^{4-6}$. Esta segunda especie es la causante de las formas cutáneas, al ser sus larvas endoparásitos obligados del tejido dérmico y subdérmico'.
Resulta muy práctico para el clínico clasificarlas según el órgano o tejido parasitado: miasis cutáneas, cavitarias o intestinales. Por su parte, las miasis cutáneas incluyen las afecciones de la piel y el tejido celular subcutáneo provocando tres formas clínicas: forma forunculoide, lineal rampante y subcutánea con tumores ambulatorios ${ }^{2,7}$.

Como medida preventiva, es importante reducir la exposición a la mosca mediante mosquiteros protectores y evitar el contacto con terrenos frecuentados por animales domésticos. Debe tenderse la ropa al sol y en plano vertical, o en recintos cerrados sin presencia de moscas. Se recomienda realizar un cuidadoso planchado de la ropa por ambos lados antes de guardarla ${ }^{8}$.

El tratamiento puede ser hospitalario o ambulatorio y, dependiendo de la gravedad de la infección, es relativamente sencillo. Basta impedir el requerimiento de oxígeno de las larvas, aplicando sustancias tales como: vaselina, parafina líquida, aceite, carne cruda, goma de mascar, cinta adhesiva, yeso pegajoso, esmalte para uñas, cera de abeja o, incluso, tocino o una pasta de tabaco. De esta manera se las obliga a salir o acercarse a la superficie para su extracción. A veces hay que inyectar un anestésico en la piel, realizar una pequeña incisión y sacar la larva con una pinza.

Se ha usado el gran poder insecticida y vermicida del ivermectin en forma oral 0,2 miligramos por kilogramo de peso, administrada en dosis única o en aplicación 
tópica en loción o ungüento ${ }^{8,9}$. Se ha utilizado el tratamiento empírico con antiparasitarios como mebendazol, tinidazol o tiabendazol en las dosis habituales.

El objetivo de este trabajo es realizar una revisión y presentación del primer caso diagnosticado en el Servicio de Medicina de la Policlínica Virgen de Guadalupe de Mongomo (Guinea Ecuatorial) para contribuir al conocimiento de la entidad.

\section{Caso clínico}

\section{Antecedentes y enfermedad actual}

Paciente de 27 años, de origen asiático, trabajador de un área constructiva, que llegó al país varias semanas antes. Consulta por un cuadro iniciado hace tres días: lesiones en piel (forúnculos) que aumentaban de tamaño y se hicieron dolorosas, en región posterior de antebrazos y espalda con secreción serosanguinolentas. Inicialmente se consideraron lesiones por varicela, y se le envió a la consulta de Medicina Interna. Se confirmó que había usado ropa de trabajo sin la debida limpieza higiénica y sanitaria.

\section{Exploración física}

TA: 140/90, Fc: 57 Ipm; temperatura: 36 C; Sat: O, 97\%. Mucosas: normales y húmedas. La auscultación cardiorespiratoria fue normal. Abdomen: sin dolor sin tumores ni visceromegalias. Tejido subcutáneo: no infiltrado. Nervioso: consciente y orientado. Sin déficit motor ni sensitivo. Sin signos meníngeos.

La piel mostraba lesiones de aspecto forunculoide, redondeadas y eritematosas, de tamaño variable, que hacían relieve sobre la superficie cutánea con centro blanquecino-amarillento, induradas al tacto y con secreciones serosanguinolentas; aumentaron en número hasta llegar aproximadamente a más de 30 lesiones, con sensación dolorosa y gran ansiedad. Al observarse detenidamente las lesiones con un objetivo de aumento, se detecta la presencia de un punto blanquecino y móvil semejante a un gusano. Se presumió el diagnóstico y se le ingresó (Figura 1).

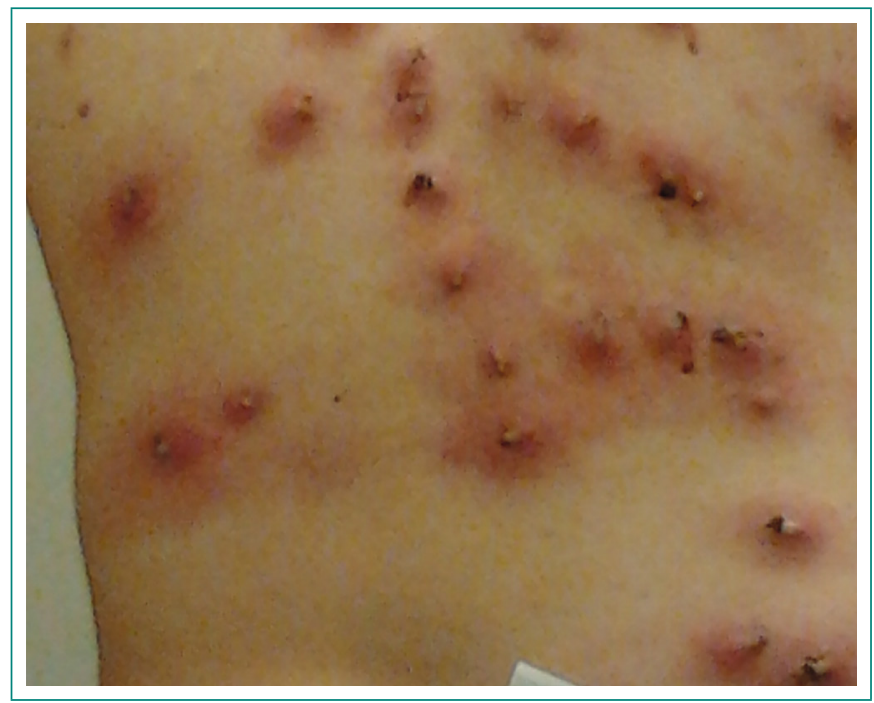

Figura 1. Lesiones cutáneas al ingreso.

\section{Pruebas complementarias}

Hemograma, leucocitos con diferencial, plaquetas y glicemia normales. Gota gruesa para malaria negativa.

\section{Evolución}

Se inició tratamiento analgésico, hidratación, antibioticoterapia sistémica (amoxicilina/ácido clavulónico: 875/125 mg) 3 veces al día; tratamiento local con pomada antibiótica vaselinada (mupirocina) y albendazol 100 mg oral cada 12 horas durante 5 días.

A los pocos días, se comenzaron a extraer las primeras larvas hasta un total de 35, identificadas como larvas de Cordylobia antropophaga. Mejoró el cuadro clínico con recuperación paulatina de las lesiones cutáneas (Figura 2).

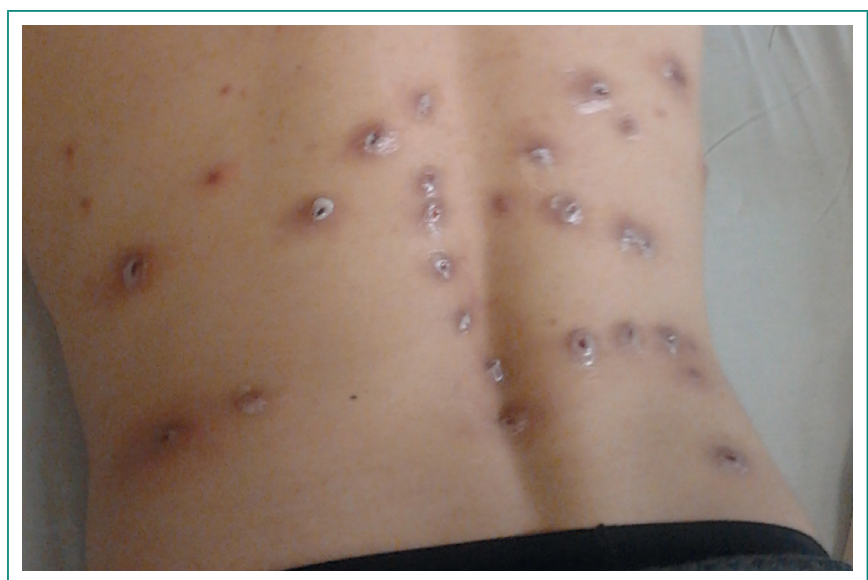

Figura 2. Lesiones cutáneas al egreso.

Fue dado de alta 7 días después con la orientación de mantener medidas higiénicas sanitarias tanto en la ropa como con sus artículos personales. Tres semanas tras el alta, acudió a consulta y ya solo quedaban secuelas de las lesiones cicatrizales (Figura 3).

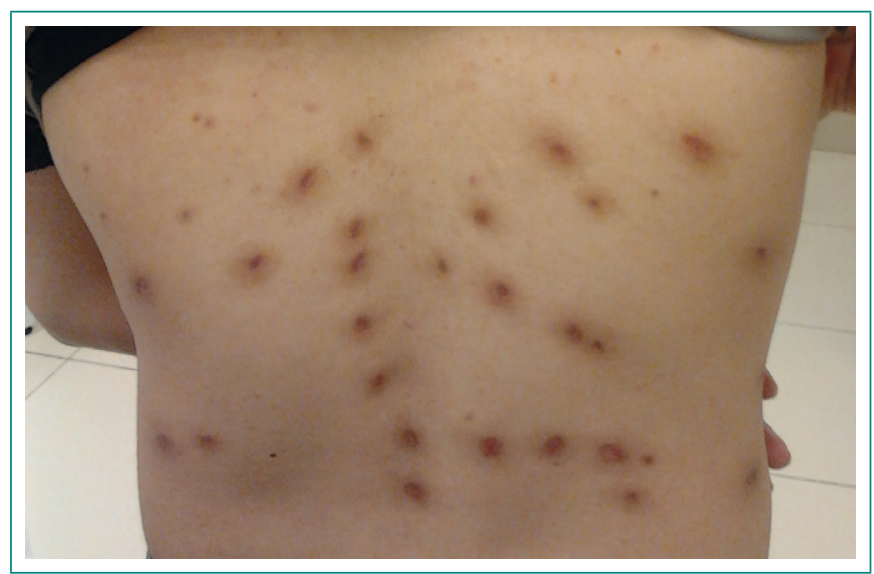

Figura 3. Lesiones cutáneas un mes después del tratamiento.

\section{Diagnóstico}

Miasis cutánea forunculoide por Cordylobia anthropophaga.

\section{Discusión y conclusión}

Las miasis son más frecuentes en países en vías de desarrollo donde las condiciones higiénicas sanitarias ${ }^{9}$ son muy deficientes y existe el agente transmisor. 
El incremento progresivo en el número de personas que viajan a los países tropicales ha hecho que estas enfermedades constituyan una parte sustancial de los diagnósticos a los que se enfrentan los servicios médicos ${ }^{10}$.

La infrecuente casuística en los países de donde proceden muchos de los médicos especialistas que van a trabajar a áreas endémicas hace que no se plantee este diagnóstico como causa de las lesiones cutáneas. Por esta razón, se notifican pocos $\operatorname{casos}^{6}$.

En Guinea Ecuatorial abundan estos dípteros, que, en su fase adulta, se alimentan de jugos vegetales, mientras que sus larvas parasitan al hombre y a otros vertebrados ${ }^{10}$. Se producen con más frecuencia en los meses de verano y se asocian a factores de riesgo, tales como edad avanzada, vagabundos, alcoholismo, enfermedad vascular periférica o diabetes mellitus, clase social baja y falta de higiene ${ }^{9}$.

Los casos de miasis cutánea no tienen la gravedad de otras enfermedades endémicas existentes, pero es necesario su conocimiento desde el punto de vista preventivo, diagnóstico y terapéutico.

Un rápido y correcto diagnóstico permite instaurar las medidas terapéuticas oportunas en cada caso y tranquilizar al paciente ya que, aunque puede ser alarmante, el cuadro clínico es habitualmente autolimitado, y normalmente con pocas secuelas ${ }^{10}$.

Nuestro paciente presentaba lesiones cutáneas forunculoides y el factor de riesgo fue la falta de medidas higiénicas adecuadas al usar ropa de trabajo ${ }^{11}$. Aunque se han propuesto varias técnicas para extraer las larvas, entre otras la remoción mecánica o forzar la migración de las larvas hacia la superficie ${ }^{12}$, en este caso fue impedir la respiración de la larva para obligarla a salir y, posteriormente, extraerla de forma mecánica con una pinza por el personal de enfermería. Además se utilizó un tratamiento antiparasitario y antibiótico para la sobreinfección de las lesiones. El paciente sufrió un cuadro de ansiedad al ver su cuerpo, literalmente, infectado por decenas de larvas, lo cual originó la necesidad de psicoterapia de apoyo por los médicos de asistencia ${ }^{10}$.

\section{Bibliografía}

1. Durán Marrero K, Montenegro Valera I, Uribe-Echeverría Delgado Al. Miasis cutánea forunculoide: un caso diagnosticado en Cuba. Rev Cubana Med Gen Integr [Internet] 2006 julio-setiembre. (Acceso 20 junio 2019); 22 (3). Accesible en: http://scielo.sld.cu/scielo.php? script=sci_arttext\&pid=S086421252006000300006\&lng=es\&nrm= iso\&tlng=es.

2. Matos Figueredo FN, Cortés Ros OM, Rodríguez Corría N. Lepra lepromatosa y miasis cutánea forunculosa. Presentación de un caso. Medisur [Internet] 2012 setiembre-octubre. (Acceso 20 junio 2019); 10 (5). Accesible en: http://scielo.sld.cu/scielo.php?script=sci_arttext \&pid=S1727897X2012000500016\&nrm=isso.

3. Solomon M, Lachish T, Schwartz E. Cutaneous Myiasis. Review. Curr Infect Dis. Rep. 2016; 18(9): 28. doi: 10.1007/s1 1908-016-0537-6.

4. Dueñas Moreira O, Gámez Sánchez D, González Guerra QM. Miasis cutánea en un anciano de una comunidad rural ecuatoriana. MEDISAN [Internet] Santiago de Cuba. 2017 mayo. (Acceso 20 junio 2019); 21 (5). Disponible en: http://scielo.sld.cu/scielo.php?Script =sci_ arttext\&pid=S1029-30192017000500012\&lng=es\&nrm=iso\&tlng =es.

5. Martínez-González MI, Heras-González S, Fatsini-Blanch V, Martínez AM. Miasis forunculoide, a propósito de un caso importado. SEMERGEN. 2017; 43 (7): 528-9. doi: 10.1016/j.semerg.2017.01.016.

6. Kuria SK, Oyedeji AO. Human myiasis cases originating and reported in Africa for the last two decades (1998-2018): A review. Acta Tropica. 15 Jun 2020; 210: 105590. doi: 10.1016/j. actatropica.2020.105590.
7. Lachish T, Marhoom E, Mumcuoglu KY, Tandlich M, Schwartz E. Myiasis in Travelers. Journal of Travel Medicine. 1 July 2015; 22(4): 232-36. https://doi. org/10.1111/jtm.12203.

8. de Hollanda Ramírez AM, Silva Rodríguez AR, Zaracho G. Ivermectina en el tratamiento de la Miasis Humana. An Fac Cienc Méd. (Asunción). [Internet] 2005 august. (Acceso 20 junio 2019); 38(3). Accesible en : http://scielo.iics. una.py/scielo.php?script=sci_arttext\&pid=S1816-89492005000200010.

9. Bollea Garlatti ML, Martínez Font A, Soledad Vacas A, Pizarro Guevara G. Miasis: diferentes formas de presentación clínica. Rev. Hosp. Ital. B.Aires 2017; 37(1): 34-38. Accesible en: https://www1.hospitalitaliano.org.ar/multimedia/archivos/noticias_attachs/47/documentos/31092_34-38-Garlatti-D. pdf.

10. Alkorta Gurrutxaga M, Beristaín Rementeria X, Cilla Eguiluz G, Tuneu Valls A, Zubizarreta Salvador J. Miasis cutánea por Cordylobia anthropophaga. Rev Esp Salud Pública. [Internet] Madrid. 2001 enero-febrero. (Acceso 20 junio 2019); 75(1). Accesible en: http://scielo.isciii. es/scielo.php?script= sci_artte xt\&pid=S1135-57272001000100004.

11. Manrique A, Manrique D, Catacora J. Miasis cutánea: reporte de un caso y revisión de la Literatura. Folia dermatol Perú. 2009; 20 (1): 23-6. Accesible en: https://www.yumpu.com/es/document/read/34851527/miasis-cutaneareporte-de-un-caso-y-revisian-de-la-literatura.

12. Yasukawa K, Dass K. Myiasis due to Cordylobia anthropophaga. Am J Trop Med Hyg. 2020 Feb; 102(2): 251. doi: 10.4269/ajtmh.19-0579. 